MARKERS OF DISEASE EVOLUTION: THE CASE OF TUBERCULOSIS

BU-1534 -M

October, 2000

\author{
Juan P. Aparicio \\ Angel F. Capurro \\ and \\ Carlos Castillo-Chavez
}

Keywords: population dynamics, epidemic models, TB, disease evolution, industrial revolution.

\begin{abstract}
Abrupt changes in environmental conditions - broadly understood to include demographic and social dynamics - can seriously impact the local or global dynamics of a population. These changes may occur over relatively short evolutionary time scales. In this note, we illustrate the potential impact of such shifts on the evolution of virulence in tuberculosis.
\end{abstract}




\title{
Markers of disease evolution: the case of tuberculosis.
}

\author{
Juan P. Aparicio ${ }^{1,2}$, Angel F. Capurro ${ }^{2,3}$ and Carlos Castillo-Chavez ${ }^{1,4}$ \\ ${ }^{1}$ Department of Biometrics \& Mathematical and Theoretical Biology Institute, \\ Cornell University, 431 Warren Hall, Ithaca, NY 14853-7801, USA \\ ${ }^{2}$ Departamento de Investigación, Universidad de Belgrano-CONICET \\ Zabala 1851, Piso 12, 1426 Buenos Aires, Argentina. \\ ${ }^{3}$ Laboratorio de Ecología, Universidad Nacional de Luján-CONICET, \\ Ruta 5 y 7, 6700 Luján, Argentina. \\ ${ }^{4}$ Department of Theoretical and Applied Mechanics \\ Cornell University, 317 Kimball Hall, Ithaca, NY 14853-1503, USA
}

October 10,2000

\begin{abstract}
Abrupt changes in environmental conditions-broadly understood to include demographic and social dynamics- can seriously impact the local or global dynamics of a population. These changes may occur over relatively short evolutionary time scales. In this note, we illustrate the potential impact of such shifts on the evolution of virulence in tuberculosis.

Key words: population dynamics, epidemic models, tuberculosis, disease evolution, Industrial Revolution

\section{Introduction}

Dramatic shifts in population structure and radical changes in living conditions took place after the Industrial Revolution. Massive migration to cities, the growth, birth and development of urban centers produced irreversible changes in the social landscape. This rapid process of urbanization increased opportunities for disease spread and evolution. Tuberculosis (TB), a communicable disease that thrives in urban settings, was no exception. Data indirectly support our hypothesis that rapid changes in the average standard of living drastically reduced the risk
\end{abstract}

of TB progression and, in the process, impacted its long-term transmission dynamics. In this note, we quantify the importance of this window in time on the evolution of TB virulence.

Most infected individuals with Mycobacterium tuberculosis do not become infectious during their lifespan. Progression rates to active-TB (the infectious disease stage) are not uniform and show a strong correlation with the average community standard of living (Bloch et al. 1989, Felton and Ford 1993, Mangtani et al. 1995, Hawker et al. 1999). Prior and throughout the nineteenth century, tuberculosis was one of the main causes of mortality. In fact, during this period of time, tuberculosis was responsible for about a quarter of all reported deaths. Yet, progression from TB-infection (latent stage) to active-TB was unlikely.

TB death-rates data show a steep and sustained decline that began at least a century prior to the effective introduction of antibiotics in 1950. The causes behind this abrupt and well-documented decline are still a matter of controversy. This reduction in TB-virulence is supported (according to some) from the 'observed' reduction on the average infection transmission per case. Others have claimed that re- 
duced virulence is mostly the result of a reduction on the risk of progression to active-TB (McKewon and Record 1962). For recent reviews see Daniel (1997) and Barnes (1995).

Reduction in the likelihood of TB-transmission has been achieved through the successful implementation of public health measures-including the practice of isolating active cases and the widespread availability of effective treatment in the past fifty years. However, these measures can hardly account for the timing and magnitude of the observed reductions. Most secondary infections produced by a source case typically take place before diagnosis, that is, within the first months of infectiousness (Daniel 1997). Forces that support an increase in the likelihood of TB transmission have actually grown with urbanization. Contact rates, a function of population density, are higher in urban settings. Hence, increase contact rates enhance the likelihood of TB-transmission (the 1919 influen $\%$ a pandemic provides an excellent example of the role of contact rates). Yet, the number of active-TB cases, has gone down. Hence, the dramatic changes on TB incidence (and prevalence) is likely to be the result of sudden reductions on average progression rates (a reduction in virulence). Progression rates have slowed down to the point that strong density-dependent increases on contact rates, in urban environments, have not been able to reverse this downward trend. Such reduction in progression rates must be the result of a combination of factors including: better average nutrition; better health practices; host evolution (Stead and Bates 1996) and, in general, a substantially improved average standard of living.

We introduce a model with density and timedependent parameters that highlight the impact of abrupt reductions in the risk of developing active-TB, over the past two centuries, on TB dynamics. The period of time of this dramatic reduction in risk must match the period of time when dramatic changes in life-expectancy at birth occurred if our model is to fit historical incidence of active-TB data. Hence, both major shifts (standard of living and TB progression) had to take place during the century that followed the start of the Industrial Revolution.

\section{The model}

TB is an airborne disease with a latent (noninfectious) period of months or decades. Most infected individuals never develop active-TB. Those who develop active-TB, generally do it during the first years following infection. Active-TB typically surfaces within ten years after infection (Styblo 1991). Infected individuals, past this high risk period, may still develop active-TB but if they do then it is likely to be caused by endogenous re-activation or re-infection.

A simple $S E I R$-model is used to match observed TB-dynamics with the support of epidemiological and demographical data from the USA. Individuals are born into the susceptible class $S$ at the rate $b$ and die at the rate $\mu$. Birth and death rates are estimated from census data (U. S. Bureau of the Census 1996). Density-dependent birth and time-dependent mortality rates are used even though time-dependent birth and death rates suffice. The mortality rate is assumed to be inversely related to life-expectancy at birth, an assumption that overestimates observed mortality rates. We keep it because it captures the main aspects of the time-evolution of the overall death rate.

The following parametric model

$$
\tau=\tau_{f}+\frac{\left(\tau_{0}-\tau_{f}\right)}{1+\exp \left[\left(t-t_{1 / 2}\right) / \Delta\right]}
$$

for the time evolution of the average life-expectancy at birth is used to fit the data. The parameters $\tau_{0}$ and $\tau_{f}$ model asymptotic values; $t_{1 / 2}$ denotes the time at which life-expectancy at birth reaches its half value, $\tau=\left(\tau_{f}+\tau_{0}\right) / 2$; and, $\Delta$ is a shape parameter-the width of the sigmoid shape function. Parameter estimates are obtained form best fit to historical data ( $\mathrm{C}$. S. Bureau of the Census 1975; see Fig. 1a). The time-evolution of the census data for the USA population $(N)$ is fitted using a logistic growth model (see Fig. 2b). Estimates of the intrinsic growth rate $(a)$ and the carrying capacity $(K)$ are obtained from the best fit to this logistic model.

Each infectious individual ( $I$ population) during his/her infectious period can produce a maximum of $Q_{0}$ secondary infections. The actual number, in a 
homogeneous mixing model, must be reduced by the factor $S / N$, the susceptible proportion.

Infection with Mycobacterium tuberculosis is likely to be for life for most individuals. Individuals who do not develop active-TB, within the first years following infection are at a low risk of developing the disease (exceptions would include HIV or other immunecompromised individuals). Re-infections may trigger TB-activation and recovered individuals may still develop active-TB again (TB-relapse). We assume that all progressions to active-TB occurs within the first years after infection, that is, we disregard re-infection and TB relapse as their incorporation, unnecessarily complicates our message. This is justified since their explicit incorporation does not seem to change the nature of the results (work in progress).

TB transmission dynamics are modeled as follows: infected individuals spend $1 / \alpha$ time (around 10 years) in the latent class $(E)$; progression to active-TB is modeled via a time-dependent rate $k(t)$; the fraction of infected people who develop active-TB is given by $f=k /(k+\alpha+\mu)$; latent individuals who escape progression to active-TB become recovered-immune individuals at the rate $\alpha$. A deterministic representation of this model is given by the following system of differential equations (see also Aparicio et al. 2000):

$$
\begin{gathered}
\frac{d S}{d t}=b N-\mu S-\gamma Q_{0} \frac{S}{N} I \\
\frac{d E}{d t}=\gamma Q_{0} \frac{S}{N} I-(k+\alpha+\mu) E \\
\frac{d I}{d t}=k E-\gamma I \\
\frac{d R}{d t}=r I+\alpha E-\mu R .
\end{gathered}
$$

Disease-induced mortality has been distributed among all classes since mortality data includes death by TB. The recovery rate from the infectious class is given by $r$ and $\gamma=r+\mu$. If the parameters were time-independent then one could define the basic reproductive number for model $(2-5)$ as $\mathcal{R}_{0}=Q_{0} f$.

\section{Results, discussion and con- clusions}

A useful measure of the risk of progression to activeTB is given by the fraction $f=k /(k+\alpha+\mu)$. This fraction is used to estimate the proportion of infected people who develop active-TB during their life-spans. Current estimates of this fraction are between 0.05 and 0.1 in developed countries (see Barnes 1995, and references therein). It is only naturally to assume that $f$ had a higher value in the past. This last assumption is enough to reproduce the patterns of (high) incidence of active-TB over the past 150 years!

Malnutrition (Chan et al. 1996, Raloff 1996) and other factors that weaken the body immune's response are known to increase the risk of developing active-TB. Hence, it is not a stretch to assume that increases in the average standard of living actually streghthens the average population immune-response to diseases like TB. The time evolution of average life-expectancy at birth can be a natural surrogate measure for the time evolution of the average standard of living of a population.

Data show that life-expectancy at birth varied abruptly during a one-hundred year time window (see Figure 1a). Hence, the time-evolution of $f$ is fitted to the same family of parametric models (1), that is, to

$$
f(t)=f_{f}+\frac{\left(f_{i}-f_{f}\right)}{1+\exp \left[\left(t-t_{1 / 2}\right) / \Delta\right]} .
$$

The parameter values of $t_{1 / 2}$ and $\Delta$, which determine when and how abrupt this transition was, are those values obtained from the best fit of (1) to lifeexpectancy data. Therefore, Model (6) has only two free parameters: the asymptotic values $f_{i}$ and $f_{f}$.

The maximum number of secondary infections produced per infectious individual, $Q_{0}$, has been growing with time because urban growth. Our simulations assume constant values for $Q_{0}$. The use of a wide range of values that cover a multitude of possible conservative scenarios mitigate the effect of this assumption.

Each value of $Q_{0}$ generates a pair of asymptotic values for $f_{i}$ and $f_{f}$, that correspond to the best fit to the data. Figure 2 shows TB-model solutions when $Q_{0}=8$, a low value and, $Q_{0}=20$, a high value. The 
corresponding $f_{i}$ values are 0.27 and 0.18 while those for $f_{f}$ are 0.1 and 0.045 . These sets of values are within a reasonable range of the expected values of the fraction $f$ before the twentieth century and today, respectively.

The numbers $\mathcal{R}_{0 i}=Q_{0} f_{i}$ and $\mathcal{R}_{0 f}=Q_{0} f_{f}$ provide estimates of potential pathogen reproduction before the Industrial Revolution and today, respectively. The sets of values for $Q_{0}, f_{i}$ and $f_{f}$ give rise to values for $\mathcal{R}_{0 i}$ between around 2 and 3.5 and, for $\mathcal{R}_{0 f}$ between 0.8 and 0.9 .

The period in which $f(t)$ experienced a sharp transition occurred right after the Industrial Revolution. The variation in $f(t)$ values has slowed down considerably since then. Today values obtained for the basic reproductive numbers $\left(Q_{0} f_{f}\right)$ are all less than, but close to, one in all simulated scenarios. That is, our model predicts a slow decline as well as the ultimate extinction of TB. Clearly, different asymptotic values for $f$ may be obtained from more detailed models. Population structure and other factors may indeed give rise to values of $\mathcal{R}_{\mathbf{0}}>1$. Our simple model cannot differentiate confidently between survival or ultimate extinction. Furthermore, in general $\mathcal{R}_{0}<1$ does not always imply extinction. The addition of exogeneous re-infection illustrates this possibility in the case of TB. Hence, exogeneous re-infection highlights the criticality of 'initial' conditions and hence, the potential fundamental role of immigration on disease persistence and re-emergence (Feng et al. 2000).

Present values for the risk $f$ also vary across communities. Slowly growing incidence of active-TB has been observed in other regions of the world (Eastern Europe and Southern Asia, for example). These trends could be the result of parameter stabilization at values for which $\mathcal{R}_{0}>1$.

Tuberculosis is not the only disease whose dynamics have been affected by improving standards of living. The transmission dynamics of diseases like cholera and influenza, to name a few, have also been strongly influenced by sociological changes that include but are not limited to improved sanitary conditions, development of vaccines, generalized used and availability of antibiotics, and the massive use of ground and air-transportation.

Abrupt sociological changes that impact the social
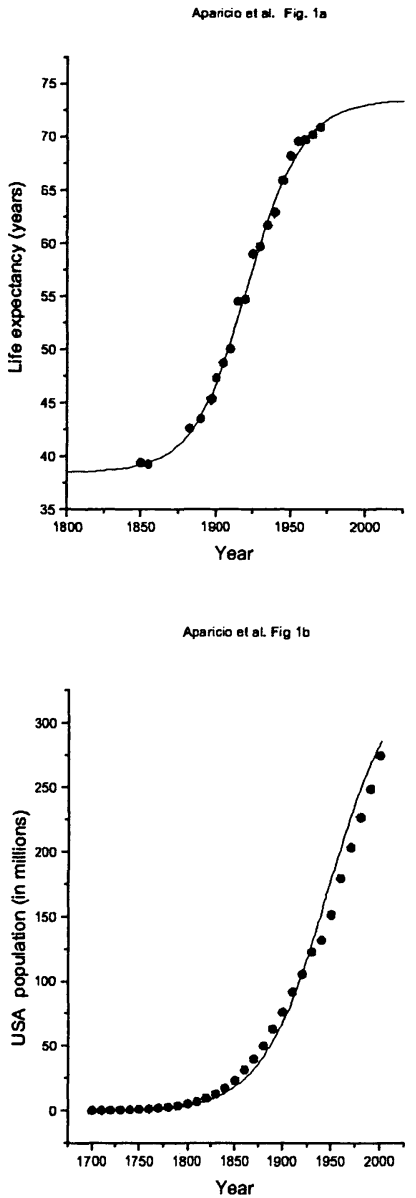

Figure 1: a) Observed average life expectancy at birth $(\bullet)$ and its best fit (continuous line) using Expression (1). Fitted parameter values are $t_{1 / 2}=$ 1921.3yr, $\Delta=18.445 y r, \tau_{i}=38.5 y r$ and $\tau_{f}=$ 73.5yr. b) Observed population growth (•) contrasted with population growth obtained with model (2-5) driven by mortality rate $\mu(t)=1 / \tau(t)$ and birth rate $b=a N(1-N / K)+\mu N$. Fitted parameter values are $a=0.0293528 y r^{-1}$ and $K=345 \times 10^{6}$ 


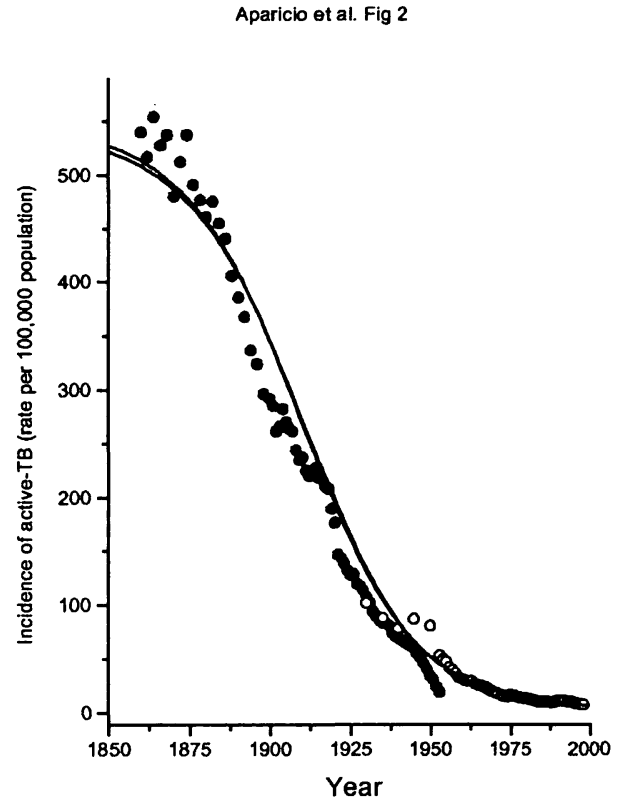

Figure 2: Observed (०), estimated (•), and model solutions (continuous line) for the incidence of activeTB (rate per 100,000 population). The observed ratio, incidence of active-TB to mortality rates, before availability of effective treatment (1930-1940) is around 1.5. Prior values for the incidence of activeTB rate are estimated to be $50 \%$ higher than the corresponding TB mortality rates (Bureau of the Census 1975). Parameter values are: $r=0.5 y r^{-1}$, $\alpha=0.1 y r^{-1}, k(t)=\frac{(\alpha+\mu) f}{1-f}$, with $\Delta$ and $t_{1 / 2}$ as in Fig. 1b. Here we present two scenarios, $Q_{0}=8$ for which $f_{i}=0.27$ and $f_{f}=0.1$, and $Q_{0}=20$ for which $f_{i}=0.18$ and $f_{f}=0.045$. landscape and its associated social dynamics may impact disease evolution. We have argued that data supports this view in the case of tuberculosis- a slowly progressing disease.

Trends observed during such exceptional and short periods (in an evolutionary time-scale) cannot be expected to continue forever. It would be not surprising to see downward trends stabilized at levels not predicted by models-like ours-that assume downward monotone trends to asymptotic values. Re-emergence of diseases like tuberculosis may just correspond to those epidemic patterns that are observed after the impact of a deep historical transition period.

Our conclusions do not depend on the scale used (the USA population). We have repeated the analysis using data from the State of Massachusetts and the City of Boston. We suspect that TB evolution in Africa would not match our temporal window because the growth and birth of urban centers took place more recently.

\section{Acknowledgments}

This work has been partially supported by NSF and NSA grants to the Mathematical and Theoretical Biology Institute at Cornell University, the office of the Provost of Cornell University, and by INCO grant $n^{\circ} 950809$ of Commission of European Community-Directorate General XII to A.F.C.

\section{REEFERENCES}

Aparicio, J. P., Capurro, A. F. and Castillo-Chavez, C. 2000. Transmission and dynamics of tuberculosis on generalized households. - J. theor. Biol. 206: 327-341,doi:10.1006/jtbi.2000.2129.

Barnes D.S. 1995. The making of a social disease: tuberculosis in the nineteenth-century France. University of California Press, pp 5-13.

Bloch A. B., Rieder H. L., Kelly G. D., Cauthen G. M., Hayden C. H. and Snider D. E. Jr. 1989. The epidemiology of tuberculosis in the United States. - Clin. Chest.Med. 10: 297-313. 
Chan, J., Tian, Y., Tanaka, E., Tsang, M. S., Yu, K. Salgame, P. Carroll, D., Kress, Y., Teitelbaum, R. and Bloom, B. R. 1996. Effects of protein malnutrition on tuberculosisi in mice. - Proc. Natl. Acad. Sci. USA 93: 14857-14861.

Daniel, T. M. 1997. Captain of death: the story of tuberculosis - University of Rochester Press, pp 37-39.

Felton C. P. and Ford J. G. 1993. Tuberculosis in the Inner City. - In: Reichman L.B., Hershfield E. S. (eds.)Tuberculois. A Comprehensive International Approach. Marcel Dekker Inc, pp. 483-504.

Feng, Z., Castillo-Chavez, C. and Capurro, A. F. 2000. A model for tuberculosis with exogeneous reinfection. - Theor. Pop. Biol. 57: 235-247, doi:10.1006/tpbi.2000.1451.

Hawker, J. I, Bakshi, S. S., Ali, S. and Farrington, C. P. 1999. Ecologival analysis of ethnic differences in relation between tuberculosis and poverty. BMJ 319: 1031-1034.

Mangtani P., Jolley D. J., Watson J. M. and Rodrigues L. C. 1995. Socioeconomics deprivation and notification rates for tuberculosis in London during 1982-1991.- BMJ 310: 963-966.

McKewon, T. and Record, R. G. 1962. Reasons for the decline of mortality in England and Wales during the nineteenth century. - Population Studies 16: 94-122.

Raloff, J. 1996. Protein deficiency abets tuberculosis. - Science 150: 374.

Stead W. W. and Bates, J. H. 1996. Geographic and evolutionary epidemiology of tuberculosis. - In: Rom W. N. \& Garay, S. M. (eds) Tuberculosis. Little, Brown and co., pp. 77-84.

Styblo, K. 1991. Selected Papers, Epidemiology of Tuberculosis. - Royal Netherlands Tuberculosis Association 24, Sec. 5.5.
U. S. Bureau of the Census. 1996. Statistical Abstract of the United States. - See also, http://www.census.gov/population.

U. S. Bureau of the Census. 1975. Historical statistics of the United States: colonial times to 1970 Washington, D. C. Government Printing Office. 